\title{
Microarray analysis of long non-coding RNA expression in human acute rejection biopsy samples following renal transplantation
}

\author{
WENBIAO CHEN $^{1 *}$, WUJIAN PENG ${ }^{2 *}$, JIANRONG HUANG ${ }^{2}$, XIANGQI YU ${ }^{1}$, KUIBI TAN $^{4}$, \\ YUYU CHEN $^{1}$, XIAOCONG LIN ${ }^{3}$, DEHENG CHEN ${ }^{1}$ and YONG DAI ${ }^{1}$ \\ ${ }^{1}$ Second Clinical Medical College of Jinan University, Shenzhen People's Hospital, Shenzhen, Guangdong 518020; \\ ${ }^{2}$ Third People's Hospital of Shenzhen, Guangdong Medical College, Shenzhen, Guangdong 518112; \\ ${ }^{3}$ Institute of Biochemistry and Molecular Biology, Guangdong Medical College, Zhanjiang, \\ Guangdong 524000; ${ }^{4}$ Ningbo Second Hospital, Ningbo, Zhejiang 315100, P.R. China
}

Received November 30, 2013; Accepted June 10, 2014

DOI: $10.3892 / \mathrm{mmr} .2014 .2420$

\begin{abstract}
Rejection is still a major obstacle in long-term allograft survival of renal transplant recipients. Long non-coding RNAs (lncRNAs) are an important class of pervasive RNAs involved in a variety of biological functions, and which are often found to be differentially expressed between healthy and pathological conditions. The aim of this study was to compare the expression profiles of lncRNAs between samples from acute rejection following kidney transplantation and control samples. Three patients were enrolled, diagnosed by renal biopsy with acute rejection upon kidney transplantation. We used lncRNA microarrays to study the lncRNA expression profiles in the kidney biopsies of these patients and in kidneys from healthy donors. Reverse transcription-quantitative polymerase chain reaction (RT-qPCR) was used to validate the microarray results. In addition, potential functions of the identified lncRNAs were further explored by searching the UCSC, RNAdb, RefSeq and NRED databases. Five candidate lncRNAs displaying differential expression in acute rejection samples were validated by RT-qPCR. The results were in agreement with the microarray data. Among the identified lncRNAs, certain have been previously identified in relevant conditions, thereby supporting previous evidence, but certain may constitute novel biomarker candidates. This is the first report to date using lncRNA microarrays to identify unique expression signatures of acute rejection in transplant biopsies. Our data indicate that lncRNAs are potentially involved in the
\end{abstract}

Correspondence to: Dr Yong Dai, Second Clinical Medical College of Jinan University, Shenzhen People's Hospital, 1017 North Road, Shenzhen, Guangdong 518020, P.R. China

E-mail: daiyong2222@gmail.com

*Contributed equally

Key words: long non-coding RNA, microarray, acute rejection, renal transplantation pathogenesis of acute rejection. Our results may have important implications in the identification of diagnostic biomarkers, as well as in the understanding and treatment of acute rejection following renal transplantation.

\section{Introduction}

Renal transplantation is the best currently available therapy for patients with end-stage renal disease, since it results not only in better survival rates, but also, in better quality of life for the patients compared to dialysis. Rejection is still a major obstacle in long-term allograft survival in renal transplant recipients. The decrease in the incidence of clinical rejections and the increased graft survival at one year after transplantation do not improve the long-term outcome of renal transplant patients. Current rates of graft loss are in the order of $2 \%$ per year (1). Numerous risk factors are known to influence graft survival, such as the age of the recipient, its ethnicity, diabetes, delayed graft function and human leukocyte antigen mismatch $(2,3)$. Acute rejection (AR) has consistently been reported to be the most important immunologic risk factor leading to chronic allograft nephropathy (4-6), which is also the most common cause of graft loss after the first year of transplantation $(7,8)$. AR is a complex process of injury of the allograft, caused by infiltrating cells of the host immune system.

The diagnosis of acute renal allograft rejection is commonly based on the increase in the serum creatinine level, indicating renal injury. The 'gold standard' for clinical diagnosis of acute rejection in patients with kidney transplants is based on histological classification of a graft biopsy, which is costly, invasive and entails risks of complications (9-12). In order to develop the most appropriate molecular diagnostic criteria, several research groups have employed '-omics' technologies, such as transcriptomics and proteomics, expected to allow identifying novel biomarkers and unravelling the biological mechanisms underlying acute rejection following transplantation (13). In a previous study, our group identified 20 microRNAs (miRNAs) differentially expressed (DE) in acute rejection following renal transplantation. These data 
indicated that miRNAs are involved in the pathogenesis of acute rejection, and may be useful in diagnosis, treatment and prevention of acute rejection after renal transplantation (14). Unfortunately, candidates from currently employed approaches do not fulfill the criteria for clinical application (15).

Long non-coding RNAs (lncRNAs) are transcripts longer than 200 nt with little or no protein-coding capacity. LncRNAs can both up-and downregulate gene expression in eukaryotes and prokaryotes, and are essential in processes such as dosage compensation, genomic imprinting, developmental patterning and differentiation, and stress response (16-20). Moreover, genome-wide studies on embryonic stem cells, adult brain, $\mathrm{CD}^{+} \mathrm{T}$ cells, and a number of other tissues have indicated that the few lncRNAs that have been characterized to date display a diverse range of functions, expression profiles in specific cell types and localizations in specific subcellular compartments (21-23).

To date, the association between changes in expression of lncRNAs and acute rejection following renal transplantation has not been studied. In this study, we analyzed lncRNA expression in human renal allograft biopsies of acute rejection following renal transplantation using the Arraystar Human lncRNA arrays, and explored the association between lncRNAs and acute rejection upon renal transplantation.

\section{Materials and methods}

Patients and controls. We studied biopsies of three patients with acute rejection graded as IA and IB as per the Banff 97 classification (9). Renal biopsies were performed by clinical ultrasound observation using the BIOPTYo instrument (BARD, Murray Hill, NJ, USA). The samples constituted of renal cortex, obtained from renal resection operation. Diagnosis of acute rejection in all patients was confirmed by histological tests, as described in the histological analysis. The 3 samples of the control group were renal cortex obtained during resection operation of the renal tumor; the samples located far from the tumor tissue, and tissue structures appeared normal under the light microscope. All biopsies were performed at the 181st Hospital of Guangxi Military Area of PLA from 2011 to 2012. The present study was approved by the Ethics Committee of the 181st Hospital Guangxi Military Area of PLA and written informed consent was obtained from each patient.

Histological analysis. The biopsy material was immediately fixed in $10 \%$ phosphate-buffered paraformaldehyde and stored at $4^{\circ} \mathrm{C}$. Following fixation, biopsies were dehydrated through passages in solutions of increasing ethanol concentration and were embedded in EPON 812 resin (Haide Biotech Company, Beijing, China). Serial, semi-thin $(0.5-\mu \mathrm{m}$ thick) sections were performed on a Reichert Ultracut Emicrotome (Leica, Glattbrugg, Switzerland). The resin was removed by treatment of the sections with sodium methoxide prior to rehydration, and immunostaining was performed as previously described (24).

Preparation of renal tissue samples. Renal cortex pieces $\left(<0.3 \times 0.3 \times 0.3 \mathrm{~mm}^{3}\right)$ obtained from nephrectomy were immediately washed in $\mathrm{RNase}$-free $0.9 \% \mathrm{NaCl}$, and dipped in Epicentre ${ }^{\circledR}$ RNase Inhibitor solution (Illumina, Madison, WI, USA) according to the manufacturer's instructions. Following
Table I. Quantitative PCR primers.

\begin{tabular}{ll}
\hline Name & \multicolumn{1}{c}{ Primer sequence } \\
\hline$\beta$-actin & F: 5'-CCT GTA CGC CAA CAC AGT GC-3' \\
& R: 5'-ATA CTC CTG CTT GCT GAT CC-3' \\
AF113674 & F: 5'-CCC TCG TTC ACT CTT CTG-3' \\
& R: 5'-GTG GGT ATT TGC GTC TTT-3' \\
uc003wbj & F: 5'-CTG GTG GGT GAA TGG GAA GG-3' \\
& R: 5'-TTG ACA GCG GAA GTG GTT GC-3' \\
uc010ftb & F: 5'-GAC AAG GAA GCC GAG TCG TA-3' \\
& R: 5'-ACA GCT GCC CTC ATT ACT ACC-3' \\
uc001fty & F: 5'-TTT ACA GTG GGT GGG TCT-3' \\
& R: 5'-GGC TTC CTT CAA AGT TCC-3' \\
AK129917 & F: 5'-AGT CTT ATC CAC TGC CAC GG-3' \\
& R: 5'-CTC AAG CAA TCC TCC TAC CA-3'
\end{tabular}

F, forward; R, reverse.

overnight storage at $4^{\circ} \mathrm{C}$, the depressor was removed from the samples, which were stored at $-80^{\circ} \mathrm{C}$.

RNA isolation and target labeling. Total RNA was extracted using the Invitrogen ${ }^{\mathrm{TM}}$ TRIzol reagent (Thermo Fisher Scientific, Waltham, MA, USA) and the RNeasy kit (Qiagen, Hilden, Germany) following the manufacturer's instructions, including a DNase digestion step. Following measurement of the RNA concentration on the Nanodrop ND-1000 machine (NanoDrop Technologies, Wilmington, DE, USA) and denaturing gel electrophoresis, the samples were used to synthesize double-stranded cDNA using the Invitrogen ${ }^{\mathrm{TM}}$ Superscript $^{\circledR}$ Double-Stranded cDNA Synthesis kit (Thermo Fisher Scientific). cDNA synthesis was performed for $60 \mathrm{~min}$ at $37^{\circ} \mathrm{C}$. The cDNA was labeled using a biotinylated nucleotide in vitro labeling kit (Affymetrix, Santa Clara, CA, USA) and hybridized to the $12 x 135 \mathrm{~K}$ LncRNA Expression microarray using the NimbleGen Hybridization System (Roche Diagnostics, Shanghai, China).

Microarray expression analysis. The microarray used in this study is designed for global profiling of long transcripts, including lncRNAs and protein-coding mRNAs. Each transcript is represented by 1-5 unique probes, in order to improve statistical confidence. Probes for housekeeping genes and negative probes are present multiple times to ensure hybridization quality. Human lncRNAs $(n=18,534)$ in this microarray have been collected from multiple data sources, including the NCBI RefSeq database (db), the University of California, Santa Cruz (UCSC) Genome db, RNAdb (http://research.imb.uq.edu.au/ rnadb/), NRED (http:/nred.matticklab.com/cgi-bin/ncrnadb. $\mathrm{pl}$ ), and the literature. Highly similar sequences and ncRNAs $<200$ bp are excluded. Protein-coding genes $(n=18,847)$ from the NCBI RefSeq db are also contained on this array, to allow simultaneous detection of mRNAs and lncRNAs in a single experiment.

Raw data were extracted as pair files using the NimbleScan software version 2.5 (Roche Diagnostics). Quantile normalization and background correction were performed with the RMA 
Table II. Optical densities (OD) of the total RNA acute rejection (AR) and normal (healthy) control (NC) samples.

\begin{tabular}{lccccc}
\hline Sample & $\mathrm{OD}_{260}$ & $\mathrm{OD}_{280}$ & $\mathrm{OD}_{260} / \mathrm{OD}_{280}$ & $\mathrm{OD}_{260} / \mathrm{OD}_{230}$ & Total RNA concentration $(\mathrm{ng} / \mu \mathrm{l})$ \\
\hline $\mathrm{AR}$ & 39.471 & 19.803 & 1.99 & 2.27 & 1578.82 \\
$\mathrm{NC}$ & 24.335 & 12.021 & 2.02 & 2.27 & 973.39 \\
\hline
\end{tabular}

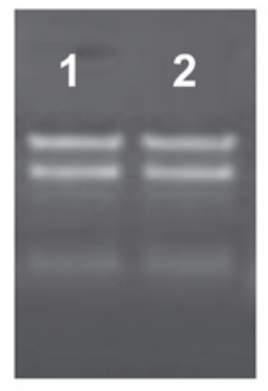

Lane 1: Total RNA of sample AR

Lane 2: Total RNA of sample NC

Figure 1. Examination of RNA integrity and genomic DNA contamination by denaturing agarose gel electrophoresis. The $28 \mathrm{~S}$ and $18 \mathrm{~S}$ ribosomal RNA should appear as fairly sharp, intense bands. The intensity of the upper band should be $\sim$ twice that of the lower band. Smaller, more diffuse bands representing low-molecular weight RNAs (tRNA and 5S ribosomal RNA) may be present. It is normal to see a diffuse smear of ethidium bromide-stained material migrating between the $18 \mathrm{~S}$ and $28 \mathrm{~S}$ ribosomal bands, probably comprised of mRNA and other heterogeneous RNA molecules. DNA contamination of the RNA preparation would manifest as a high-molecular weight smear or band migrating above the $28 \mathrm{~S}$ ribosomal RNA band. Degradation of the RNA would be reflected as a smear of ribosomal RNA bands. AR, acute rejection; NC, normal (healthy) control.

method implemented in this software. The Probe level ( ${ }^{*}$ norm RMA.pair) files and the Gene summary ( ${ }^{*}$ RMA.calls) files were created. The 2 gene summary files were imported into the GeneSpring software version 11.0 (Agilent Technologies, Inc., Santa Clara, CA, USA) for further analysis. The effect of normalization was examined on box-plots. DE lncRNAs were identified by fold-change analysis and a p-value cutoff $<0.05$.

Pathway analysis of DE RNAs. Pathway analysis was based on the pathways available at the Kyoto Encyclopedia of Genes and Genomes (KEGG) database (http://www.genome.jp/kegg/). This analysis allows to identify the biological pathways in which the differentially expressed RNAs are involved. The p-value denotes the significance of pathway enrichment based on Fisher tests: the lower the p-value, the more significantly enriched is the list of DE RNAs in this pathway. We used the recommended p-value cutoff $\leq 0.05$.

Quantitative PCR ( $q P C R)$. The qPCR reaction was carried out using $1 \mu \mathrm{l}$ (50-100 ng/ $/ \mu \mathrm{l})$ of cDNA as the template; $1 \mu \mathrm{l}$ of forward and reverse primers (listed in Table I) and $10 \mu \mathrm{l}$ of Invitrogen ${ }^{\mathrm{TM}}$ 10,000X SYBR-Green PCR Master mix (Thermo Fisher Scientific). The cDNA was serially diluted 2 -fold in nuclease-free water prior to use. The experiment was conducted in duplicate for each sample. Master mix without total RNA was prepared for all reactions, and $24 \mu \mathrm{l}$ were aliquoted into each reaction tube. The diluted cDNA was then added individually in each tube. qPCR reactions were conducted on a Rotor-Gene 3000 Real-Time PCR system (Corbett Research, New South Wales, Australia) as follows: pre-denaturation for $5 \mathrm{~min}$ at $95^{\circ} \mathrm{C} ; 40$ cycles of $10 \mathrm{sec}$ at $95^{\circ} \mathrm{C}$, $15 \mathrm{sec}$ at $58^{\circ} \mathrm{C}$, and $20 \mathrm{sec}$ at $72^{\circ} \mathrm{C}$. Melting-curve analysis was performed to determine the reaction specificity. Agarose gel electrophoresis was also performed to confirm of the size of the PCR products. The mean of the cycle threshold $(\mathrm{Ct})$ values was calculated in order to determine the linearity of the GAPDH expression level. The expression data were analyzed with the comparative CT method (25).

Statistical analysis. Signal intensities for each spot were acquired by the Axon GenePix 4000B microarray scanner (Molecular Devices, Sunnyvale, CA, USA) and further analyzed with the NimbleScan and Agilent GeneSpring software. Signal intensities for each spot were calculated by subtracting the local background (based on the median intensity of the area surrounding each spot) from the total intensity. An average value of the 5 spot replicates for each lncRNA was generated following data transformation (to convert any negative value to 0.01 ), and normalization was performed by using a "per chip normalization", in which each measurement was divided by the 50th percentile of all measurements in its array, allowing comparison among chips. In order to identify lncRNAs that characterize each group, a per-gene median normalization was performed, which normalizes the expression of every lncRNA on its median among samples.

\section{Results}

RNA quantity and quality. The quantity and quality of the acute rejection (AR) and normal (healthy) control (NC) RNA samples were assessed by gel electrophoresis (Fig. 1) and via the optical density ratio $\mathrm{OD}_{260} / \mathrm{OD}_{280}$ (Table II). These analyses confirmed that the isolated total RNA is of good quality.

LncRNA expression. To compare the expression profiles of lncRNAs in AR and NC samples, we used a custom microarray approach. Following normalization of the raw data, the differentially expressed genes or lncRNAs were identified based on fold-change differences in expression between the two groups, with a threshold value $\geq 2.0$. This analysis identified 5,339 1ncRNAs as significantly differentially expressed. Of these, 2,191 lncRNAs were found to be upregulated and 3,148 to be downregulated. The top 20 upregulated and downregulated lncRNAs in AR samples as compared to NC samples are summarized in Table III. From the DE lncRNAs, we selected, based on their fold changes and probability values, 5 for validation by RT-qPCR, which confirmed their expression profiles (Table IV). 
Table III. The top 20 up- and 20 downregulated long non-coding RNAs in acute rejection (AR) and normal (healthy) control (NC) samples.

\begin{tabular}{|c|c|c|c|c|c|}
\hline Array_id & Sequence id & NC (normalized) & AR (normalized) & Fold change & Chromosome \\
\hline \multicolumn{6}{|l|}{ Upregulated } \\
\hline ASLNC04531 & uc002rpc & 46.51560 & 17315.78000 & 372.25757 & 2 \\
\hline ASLNC09207 & uc001mng & 94.44511 & 5389.65770 & 57.06656 & 11 \\
\hline ASLNC14712 & ВC020554 & 264.48758 & 11507.56800 & 43.50892 & 2 \\
\hline ASLNC08637 & uc009xqb & 182.39748 & 6850.25240 & 37.55673 & 10 \\
\hline ASLNC13599 & ВС046920 & 163.36601 & 6082.66160 & 37.23333 & 1 \\
\hline ASLNC07985 & uc004cac & 292.56890 & 9997.94200 & 34.17294 & 9 \\
\hline ASLNC19577 & AK123269 & 149.90768 & 4837.56250 & 32.27028 & 2 \\
\hline ASLNC03665 & $\mathrm{uc} 001 \mathrm{fcl}$ & 684.48210 & 21773.67600 & 31.81043 & 1 \\
\hline ASLNC12108 & uc002lxr & 923.49524 & 29205.33600 & 31.62478 & 19 \\
\hline ASLNC21766 & AL117622 & 39.51770 & 1140.18730 & 28.85257 & 2 \\
\hline ASLNC18894 & AK095208 & 181.09515 & 5154.58400 & 28.46340 & $\mathrm{Y}$ \\
\hline ASLNC11418 & uc002gwi & 43.88539 & 1162.90340 & 26.49864 & 17 \\
\hline ASLNC03614 & uc009wpu & 119.10841 & 3144.25510 & 26.39826 & 1 \\
\hline ASLNC11432 & uc010cqs & 53.65291 & 1416.20410 & 26.39566 & 17 \\
\hline ASLNC04193 & uc001axt & 187.77946 & 4847.61100 & 25.81544 & 1 \\
\hline ASLNC12713 & uc002ywy & 33.03370 & 851.20294 & 25.76771 & 21 \\
\hline ASLNC00922 & NR_024418 & 84.38660 & 2173.93480 & 25.76161 & 5 \\
\hline ASLNC00559 & NR_003377 & 66.64611 & 1700.32530 & 25.51274 & 1 \\
\hline ASLNC17358 & AK026561 & 26.36899 & 580.09990 & 21.99931 & 4 \\
\hline ASLNC14352 & AL049227 & 38.68229 & 778.73160 & 20.13147 & 5 \\
\hline \multicolumn{6}{|l|}{ Downregulated } \\
\hline ASLNC06892 & uc003wcs & 12141.12600 & 205.09033 & 59.19891 & 7 \\
\hline ASLNC02456 & ASO3568 & 2672.73120 & 60.76619 & 43.98385 & 9 \\
\hline ASLNC08095 & uc003zfx & 2563.60520 & 63.50388 & 40.36926 & 9 \\
\hline ASLNC01268 & NR_015423 & 1845.16000 & 46.51090 & 39.67156 & 9 \\
\hline ASLNC10347 & uc010akv & 4372.90000 & 138.60800 & 31.54868 & 14 \\
\hline ASLNC19249 & AK097316 & 9239.31800 & 325.60782 & 28.37560 & 16 \\
\hline ASLNC07072 & uc003syy & 1711.72670 & 65.63537 & 26.07933 & 7 \\
\hline ASLNC01067 & NR_015356 & 2245.38570 & 87.05270 & 25.79340 & 7 \\
\hline ASLNC04837 & uc002tjx & 4122.41650 & 160.13051 & 25.74410 & 2 \\
\hline ASLNC22002 & AY343893 & 4541.71400 & 195.93309 & 23.17992 & 9 \\
\hline ASLNC08815 & uc001pyd & 845.93524 & 45.35469 & 18.65154 & 11 \\
\hline ASLNC17495 & AK054638 & 676.56573 & 40.45289 & 16.72478 & 8 \\
\hline ASLNC01430 & NR_024430 & 766.93530 & 53.67410 & 14.28874 & 11 \\
\hline ASLNC21970 & AY129027 & 2840.09330 & 204.75237 & 13.87087 & 9 \\
\hline ASLNC12665 & uc002zic & 15624.00400 & 1273.09390 & 12.27246 & 21 \\
\hline ASLNC12607 & uc010gqe & 16797.70300 & 1392.48970 & 12.06307 & 21 \\
\hline ASLNC00493 & NR_002946 & 4926.25100 & 428.81802 & 11.48797 & 1 \\
\hline ASLNC10383 & uc001vvi & 1599.94170 & 140.93541 & 11.35230 & 14 \\
\hline ASLNC13003 & uc002zpx & 21649.31600 & 2080.54540 & 10.40559 & 22 \\
\hline ASLNC22440 & ВC023609 & 5342.29740 & 516.37445 & 10.34578 & 10 \\
\hline
\end{tabular}

Biological pathways identified in acute rejection patients. Pathway analysis was performed next; this is a type of functional analysis mapping genes to pathways, e.g., from KEGG, followed by statistical significance tests of enrichment.
As shown in Table V, 21 pathways were significantly $(\mathrm{P}<0.05)$ enriched among the DE lncRNAs, including a number of immune-response pathways (IL2-mediated signaling events, IL6, B cell survival pathway, and $\mathrm{TNF} \alpha / \mathrm{NF}-\kappa \mathrm{B})$. These results 
Table IV. Reverse transcription-quantitative PCR data on expression of long non-coding RNAs (lncRNAs) in acute rejection (AR) and normal (healthy) control (NC) samples.

\begin{tabular}{lccccc}
\hline LncRNA & AR & NC & AR/ $\beta$-actin & NC/ $\beta$-actin & AR/NC ratio \\
\hline AF113674 & $4.28 \mathrm{E}-04$ & $9.47 \mathrm{E}-04$ & $7.07 \mathrm{E}-01$ & $1.31 \mathrm{E}+00$ & $3.98 \mathrm{E}-01$ \\
uc003wbj & $3.42 \mathrm{E}-04$ & $2.87 \mathrm{E}-04$ & $5.65 \mathrm{E}-01$ & $4.24 \mathrm{E}+00$ & 1.410 \\
uc010ftb & $4.33 \mathrm{E}-03$ & $3.06 \mathrm{E}-03$ & $7.15 \mathrm{E}+00$ & $3.73 \mathrm{E}-01$ & 1.686 \\
uc001fty & $1.79 \mathrm{E}-04$ & $2.67 \mathrm{E}-04$ & $2.95 \mathrm{E}-01$ & $1.58 \mathrm{E}-02$ & 0.790 \\
AK129917 & $9.22 \mathrm{E}-05$ & $1.14 \mathrm{E}-05$ & $1.52 \mathrm{E}-01$ & & 9.620 \\
$\beta$-actin & $6.05 \mathrm{E}-04$ & $7.21 \mathrm{E}-04$ & & & \\
\hline
\end{tabular}

Table V. The top 21 significantly enriched Kyoto Encyclopedia of Genes and Genomes pathways for the differentially expressed long non-coding RNAs (DE lncRNAs).

\begin{tabular}{|c|c|c|c|c|}
\hline Pathway name & $\begin{array}{l}\text { No. of } \\
\text { DE lncRNAs }\end{array}$ & $\begin{array}{c}\text { No. of } \\
\text { genes in pathway }\end{array}$ & $\begin{array}{l}\text { No. of DE lncRNAs } \\
\text { in pathway }\end{array}$ & P-value \\
\hline IL2-mediated signaling events & 179 & 50 & 27 & $1.80 \mathrm{E}-02$ \\
\hline IL6 & 66 & 48 & 27 & $9.11 \mathrm{E}-03$ \\
\hline ERK-PI3K (collagen) signaling & 36 & 24 & 16 & 4.72E-03 \\
\hline Alanine and aspartate metabolism & 76 & 22 & 15 & 4.50E-03 \\
\hline $\begin{array}{l}\text { Lissencephaly gene (lis1) in neuronal } \\
\text { migration and development }\end{array}$ & 18 & 11 & 9 & 4.30E-03 \\
\hline Cytochrome p450 & 54 & 6 & 6 & $3.21 \mathrm{E}-03$ \\
\hline Phase 1 functionalization & 87 & 6 & 6 & $3.21 \mathrm{E}-03$ \\
\hline Cyanoamino acid metabolism & 27 & 6 & 6 & $3.21 \mathrm{E}-03$ \\
\hline FGF signaling pathway & 78 & 29 & 19 & $2.83 \mathrm{E}-03$ \\
\hline Antiapoptotic pathway & 19 & 14 & 11 & $2.67 \mathrm{E}-03$ \\
\hline Metabolism of amino acids & 297 & 38 & 24 & $1.73 \mathrm{E}-03$ \\
\hline $\begin{array}{l}\text { FOXA } 2 \text { and FOXA } 3 \text { transcription } \\
\text { factor networks }\end{array}$ & 56 & 46 & 28 & $1.67 \mathrm{E}-03$ \\
\hline FOXA transcription factor networks & 103 & 75 & 42 & $1.48 \mathrm{E}-03$ \\
\hline B cell survival pathway & 13 & 7 & 7 & $1.23 \mathrm{E}-03$ \\
\hline Granzyme a mediated apoptosis pathway & 12 & 7 & 7 & $1.23 \mathrm{E}-03$ \\
\hline p75(NTR)-mediated signaling & 293 & 83 & 46 & $1.21 \mathrm{E}-03$ \\
\hline $\begin{array}{l}\text { Proteogylcan syndecan-mediated } \\
\text { signaling events }\end{array}$ & 306 & 104 & 56 & $9.75 \mathrm{E}-04$ \\
\hline $\begin{array}{l}\text { Neurotrophic factor-mediated Trk } \\
\text { receptor signaling }\end{array}$ & 160 & 56 & 34 & $5.90 \mathrm{E}-04$ \\
\hline IL3 & 76 & 62 & 37 & 5.53E-04 \\
\hline Syndecan-2-mediated signaling events & 130 & 41 & 28 & $1.01 \mathrm{E}-04$ \\
\hline $\mathrm{TNF} \alpha / \mathrm{NF}-\kappa \mathrm{B}$ & 214 & 165 & 93 & $2.06 \mathrm{E}-06$ \\
\hline
\end{tabular}

suggest that alterations in expression of a limited number of genes may affect the regulation of numerous immune response and inflammatory pathways that are common to acute rejection.

\section{Discussion}

High-density microarray technology provides a means to simultaneously measure the differential expression of hundreds to thousands of genes. The ability to measure the expression of IncRNAs in samples of early acute rejection following kidney transplantation allows to identify several biomarkers that have the potential to directly impact current clinical practices. In the present study, we took advantage of the high-throughput nature of the Arraystar Human lncRNA array to identify and characterize lncRNAs differentially expressed between acute rejection following renal transplantation and healthy control tissues. This study identified 5,339 DE lncRNAs, of which 3,148 were downregulated and 2,191 were upregulated in AR compared to NC samples. From the functional analysis of these DE lncRNAs using KEGG pathway enrichment 
analysis, we conclude that acute rejection is associated with immune activation and inflammation. A number of studies have investigated the expression of immune activation genes in allograft biopsies. Interleukin (IL)-7, IL-8, IL-10, IL-15 and interferon; T-cell receptor (TCR) variable regions; cytotoxic T lymphocyte effector molecules, such as perforin, granzyme B, Fas and Fas ligand; and the CD40 ligand have all been identified as upregulated in acutely rejecting allograft biopsy tissues $(13,26)$. As immune cells, such as T cells, differentiate, gene expression is affected by the combination of epigenetic alterations in DNA methylation, chromatin structure and locus accessibility (27). The roles of lncRNAs in gene and epigenetic regulation are well documented (28). The finding of the present study that thousands of lncRNAs are expressed in acute renal rejection is not surprising. In addition, the presented data are derived from only three patients.

The function of only a minority of lncRNAs is documented at present. Therefore, we studied the potential association between acute rejection and lncRNAs in order to gain new insights into the pathogenesis of acute rejection and potentially identify novel biomarkers, which are aspects that have not been previously studied. From the DE IncRNAs identified herein, we selected 5 based on their expression fold changes and probability values for further validation by RT-qPCR experiments, and further investigated their functions in the literature.

The 1ncRNA uc001fty locates at the intron of the $\mathrm{C}$-reactive protein-coding gene $(C R P)$. LncRNAs from introns have diverse regulatory functions, such as acting as precursors of shorter RNAs, protein-coding RNA stabilization, control of gene expression, and regulation of alternative splicing in protein-coding RNA (29). The uc001fty belongs to the pentaxin family. It is involved in several host defense-related functions based on its ability to recognize foreign pathogens and host damaged cells and to initiate their elimination by interacting with humoral and cellular effector systems in the blood. In our study, we observed downregulation in both uc001fty and CRP gene expression. T cells play a key role in the alloimmune response, thus it is not surprising that TCR active-chain expression was upregulated in all acute rejection samples (data not shown), as previously shown by other groups (7-9). The uc003wbj, corresponding to the Homo sapiens mRNA for the T-cell receptor $\beta$ chain, was found at relatively high expression level in the acute rejection samples, which may be related with inflammatory reactions occurring during acute rejection.

Transcribed lncRNAs interact with downstream promoter regions of protein-coding genes to regulate mRNA expression (30). The lncRNA AK129917 (chr2, mRNA) locates across the transcription start site of Hsp90B. Hsp90 proteins are highly conserved molecular chaperones that have key roles in signal transduction, protein folding, protein degradation and morphology. Overexpression of Hsp90s has been reported in rat renal cortex following ischemia and in recipients with graft-versus-host disease $(31,32)$. Interestingly, we observed that AK129917 and Hsp90Bl are both upregulated in acute rejection biopsy samples, possibly through independent regulation pathways.

The lncRNA uc010ftb (chr6, UCSC) locates across the transcription start site of the caspase 10 gene (CASP10). This IncRNA encodes a protein that is a member of the cysteineaspartic acid protease (caspase) family. Sequential activation of caspases plays a central role in the execution phase of cell apoptosis. In this study, we found that CASPIO is downregulated, but the expression of uc010ftb was upregulated. From these data, we may infer that uc010ftb negatively regulates the CASP10 gene. The lncRNA AF113674 (chr19, mRNA) also locates across the transcription start site of the complement component 3 (C3). The complement system is an important contributor to both innate and adaptive immunity, ultimately forming a proteolytic cascade that sets an inflammatory reaction in motion. Innate immunity, adaptive immunity and inflammation all converge at the $\mathrm{C} 3$ convertase step, a central amplification point in the cascade (33). The activation of the complement is associated with ischemia, reperfusion and inflammation that occur during graft rejection (34). Therefore, the AF113674 lncRNA may constitute a suitable biomarker of acute rejection.

The individual lncRNA variation between patients may be difficult to detect and of no significance, since random expression changes may occur in each patient. In our study, RNAs from each group were pooled together in the same sample, so that the individual differences among subjects are reduced. The aim of this study was to reveal the relationship between acute renal rejection and lncRNAs, expecting to draw the attention of additional research groups to this subject.

In conclusion, we presented herein an analysis of lncRNA expression in control and acute rejection renal allograft biopsy samples using lncRNA microarrays. Our results indicate that certain lncRNAs show promising potential as diagnostic biomarkers and as factors involved in the pathogenesis of acute rejection. Nevertheless, it is important to note that the findings described in this study are merely a starting point for the understanding of roles that IncRNAs may play in acute renal transplant rejection. In this context, it is notable that the function of only a minority of lncRNAs is documented at present. Additional studies are needed to functionally characterize lncRNAs, including the ones identified in this study. The list of lncRNAs linked to acute rejection following renal transplantation may promote the identification of novel methods for the diagnosis, treatment and prevention of acute renal rejection, and further illuminate the mechanisms underlying the rejection of other solid organ transplants.

\section{Acknowledgements}

The authors thank the patients who participated in this study. The authors also thank Kang Chen Biotech for technical assistance. This study was supported by grants from the National Natural Science Foundation of China (no. 30972741) and the Guangxi Natural Science Foundation (no. 2012GXNSFD-A053017).

\section{References}

1. Ekberg H, Bernasconi C, Tedesco-Silva H, et al: Calcineurin inhibitor minimization in the Symphony study: observational results 3 years after transplantation. Am J Transplant 9: 1876-1885, 2009.

2. Shoskes DA and Cecka JM: Deleterious effects of delayed graft function in cadaveric renal transplant recipients independent of acute rejection. Transplantation 66: 1697-1701, 1998.

3. Hariharan S, Johnson CP, Bresnahan BA, Taranto SE, McIntosh MJ and Stablein D: Improved graft survival after renal transplantation in the United States, 1988 to 1996. N Engl J Med 342: 605-612, 2000. 
4. Meier-Kriesche HU, Ojo AO, Hanson JA, et al: Increased impact of acute rejection on chronic allograft failure in recent era. Transplantation 70: 1098-1100, 2000.

5. Paul LC: Chronic allograft nephropathy: an update. Kidney Int 56: 783-793, 1999.

6. Humar A, Hassoun A, Kandaswamy R, Payne WD, Sutherland DE and Matas AJ: Immunologic factors: the major risk for decreased long-term renal allograft survival. Transplantation 68: 1842-1846, 1999.

7. Cosio FG, Pelletier RP, Falkenhain ME, et al: Impact of acute rejection and early allograft function on renal allograft survival. Transplantation 63: 1611-1615, 1997.

8. Woodroffe R, Yao GL, Meads C, et al: Clinical and cost-effectiveness of newer immunosuppressive regimens in renal transplantation: a systematic review and modelling study. Health Technol Assess 9: 1-179, iii-iv, 2005.

9. Racusen LC, Solez K, Colvin RB, et al: The Banff 97 working classification of renal allograft pathology. Kidney Int 55: 713-723, 1999.

10. Howie AJ: Problems with BANFF. Transplantation 73 : 1383-1384, 2002.

11. Ramanathan V, Goral S and Helderman JH: Renal transplantation. Semin Nephrol 21: 213-219, 2001.

12. Girlanda R, Mannon RB and Kirk AD: Diagnostic tools for monitoring kidney transplant recipients. Semin Nephrol 27 462-478, 2007.

13. Perkins D, Verma M and Park KJ: Advances of genomic science and systems biology in renal transplantation: a review. Semin Immunopathol 33: 211-218, 2011

14. Sui W, Dai Y, Huang Y, Lan H, Yan Q and Huang H: Microarray analysis of microRNA expression in acute rejection after renal transplantation. Transpl Immunol 19: 81-85, 2008.

15. Zand MS: Immunosuppression and immune monitoring after renal transplantation. Semin Dial 18: 511-519, 2005.

16. Mercer TR, Dinger ME and Mattick JS: Long non-coding RNAs: insights into functions. Nat Rev Genet 10: 155-159, 2009.

17. Feng J, Bi C, Clark BS, Mady R, Shah P and Kohtz JD: The Evf-2 noncoding RNA is transcribed from the Dlx-5/6 ultraconserved region and functions as a Dlx-2 transcriptional coactivator. Genes Dev 20: 1470-1484, 2006

18. Yan MD, Hong CC, Lai GM, Cheng AL, Lin YW and Chuang SE: Identification and characterization of a novel gene Saf transcribed from the opposite strand of Fas. Hum Mol Genet 14: 1465-1474, 2005.

19. Wang H, Iacoangeli A, Lin D, et al: Dendritic BC1 RNA in translational control mechanisms. J Cell Biol 171: 811-821, 2005 .
20. Sleutels F, Zwart R and Barlow DP: The non-coding Air RNA is required for silencing autosomal imprinted genes. Nature 415: 810-813, 2002.

21. Mercer TR, Dinger ME, Sunkin SM, Mehler MF and Mattick JS: Specific expression of long noncoding RNAs in the mouse brain. Proc Natl Acad Sci USA 105: 716-721, 2008.

22. Dinger ME, Amaral PP, Mercer TR, et al: Long noncoding RNAs in mouse embryonic stem cell pluripotency and differentiation. Genome Res 18: 1433-1445, 2008.

23. Pang KC, Dinger ME, Mercer TR, et al: Genome-wide identification of long noncoding RNAs in $\mathrm{CD}^{+} \mathrm{T}$ cells. J Immunol 182 7738-7748, 2009.

24. Groos S, Reale E and Luciano L: Re-evaluation of epoxy resin sections for light and electron microscopic immunostaining. J Histochem Cytochem 49: 397-406, 2001.

25. Schmittgen TD and Livak KJ: Analyzing real-time PCR data by the comparative C(T) method. Nat Protoc 3: 1101-1108, 2008.

26. Sharma VK, Bologa RM, Li B, et al: Molecular executors of cell death - differential intrarenal expression of Fas ligand, Fas, granzyme $\mathrm{B}$, and perforin during acute and/or chronic rejection of human renal allografts. Transplantation 62: 1860-1866, 1996.

27. McLean AG, Hughes D, Welsh KI, et al: Patterns of graft infiltration and cytokine gene expression during the first 10 days of kidney transplantation. Transplantation 63: 374-380, 1997.

28. Wilson CB, Makar KW, Shnyreva M and Fitzpatrick DR: DNA methylation and the expanding epigenetics of $\mathrm{T}$ cell lineage commitment. Semin Immunol 17: 105-119, 2005.

29. Mattick JS, Amaral PP, Dinger ME, Mercer TR and Mehler MF RNA regulation of epigenetic processes. Bioessays 31: 51-59, 2009.

30. Louro R, Smirnova AS and Verjovski-Almeida S: Long intronic noncoding RNA transcription: expression noise or expression choice? Genomics 93: 291-298, 2009.

31. Harrison EM, Sharpe E, Bellamy CO, et al: Heat shock protein 90-binding agents protect renal cells from oxidative stress and reduce kidney ischemia-reperfusion injury. Am J Physiol Renal Physiol 295: F397-F405, 2008.

32. Goral J, Shenoy S, Mohanakumar T and Clancy J Jr: Antibodies to $70 \mathrm{kD}$ and $90 \mathrm{kD}$ heat shock proteins are associated with graft-versus-host disease in peripheral blood stem cell transplant recipients. Clin Exp Immunol 127: 553-559, 2002.

33. Markiewski MM, DeAngelis RA and Lambris JD: Complexity of complement activation in sepsis. J Cell Mol Med 12: 2245-2254, 2008.

34. Ioannou A, Dalle Lucca J and Tsokos GC: Immunopathogenesis of ischemia/reperfusion-associated tissue damage. Clin Immunol 141: 3-14, 2011. 